University of New Hampshire

University of New Hampshire Scholars' Repository

Physics Scholarship

Physics

3-2015

\title{
Van Allen Probes show that the inner radiation zone contains no MeV electrons: ECT/MagEIS data
}

\author{
J. F. Fennell \\ Aerospce Corporation \\ S. Claudepierre \\ Aerospace Corporation \\ J. B. Blake \\ Aerospace Corporation \\ T. P. O'Brien \\ Aerospace Corporation \\ J. H. Clemmons \\ Aerospace Corporation
}

See next page for additional authors

Follow this and additional works at: https://scholars.unh.edu/physics_facpub

Part of the Astrophysics and Astronomy Commons

\section{Recommended Citation \\ J. F. Fennell, S. G. Claudepierre, J. B. Blake, T. P. O’Brien, J. H. Clemmons, D. N. Baker, H. E. Spence, and G. D. Reeves, 'Van Allen Probes show that the inner radiation zone contains no MeV electrons: ECT/MagEIS data', Geophysical Research Letters, vol. 42, no. 5, pp. 1283-1289, Mar. 2015.}

This Article is brought to you for free and open access by the Physics at University of New Hampshire Scholars' Repository. It has been accepted for inclusion in Physics Scholarship by an authorized administrator of University of New Hampshire Scholars' Repository. For more information, please contact Scholarly.Communication@unh.edu. 


\section{Authors}

J. F. Fennell, S. Claudepierre, J. B. Blake, T. P. O'Brien, J. H. Clemmons, D. N. Baker, Harlan E. Spence, and Geoffrey Reeves 


\section{Geophysical Research Letters}

\section{RESEARCH LETTER}

10.1002/2014GL062874

Key Points:

- There are no significant fluxes of $\mathrm{MeV}$ electrons in the inner zone

- Previous measurements and model estimates significantly differ from current ones

- Clean electron measurements were made deep in the inner radiation zone

Supporting Information:

- Text S1 and Figures S1-S3

Correspondence to:

J. F. Fennell,

joseph.fennell@aero.org

\section{Citation:}

Fennell, J. F., S. G. Claudepierre, J. B. Blake, T. P. O'Brien, J. H. Clemmons, D. N. Baker, H. E. Spence, and G. D. Reeves (2015), Van Allen Probes show that the inner radiation zone contains no $\mathrm{MeV}$ electrons: ECT/MagEIS data, Geophys. Res. Lett., 42, 1283-1289, doi:10.1002/2014GL062874.

Received 18 DEC 2014 Accepted 29 JAN 2015 Accepted article online 2 FEB 2015 Published online 9 MAR 2015

\section{Van Allen Probes show that the inner radiation zone contains no MeV electrons: ECT/MagEIS data}

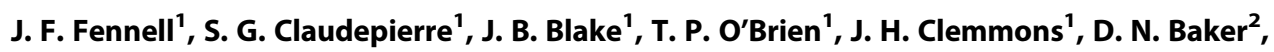 \\ H. E. Spence ${ }^{3}$, and G. D. Reeves ${ }^{4}$ \\ ${ }^{1}$ Space Science Applications Laboratory, Aerospace Corporation, El Segundo, California, USA, ${ }^{2}$ Laboratory for Atmospheric and \\ Space Physics, University of Colorado, Boulder, Colorado, USA, ${ }^{3}$ Institute for the Study of Earth, Oceans, and Space, University \\ of New Hampshire, Durham, New Hampshire, USA, ${ }^{4}$ Los Alamos National Laboratory, Los Alamos, New Mexico, USA
}

Abstract We present Van Allen Probe observations of electrons in the inner radiation zone. The measurements were made by the Energetic Particle, Composition, and Thermal Plasma/Magnetic Electron lon Spectrometer (MagEIS) sensors that were designed to measure electrons with the ability to remove unwanted signals from penetrating protons, providing clean measurements. No electrons $>900 \mathrm{keV}$ were observed with equatorial fluxes above background (i.e., $>0.1 \mathrm{el} /\left(\mathrm{cm}^{2} \mathrm{~s} \mathrm{sr} \mathrm{keV)}\right)$ in the inner zone. The observed fluxes are compared to the AE9 model and CRRES observations. Electron fluxes $<200 \mathrm{keV}$ exceeded the AE9 model $50 \%$ fluxes and were lower than the higher-energy model fluxes. Phase space density radial profiles for $1.3 \leq L^{*}<2.5$ had mostly positive gradients except near $L^{*} \sim 2.1$, where the profiles for $\mu=20-30 \mathrm{MeV} / \mathrm{G}$ were flat or slightly peaked. The major result is that MagEIS data do not show the presence of significant fluxes of MeV electrons in the inner zone while current radiation belt models and previous publications do.

\section{Introduction}

The radiation belts are generally described as having inner and outer zones or belts with a transition region or slot between them. The inner zone is dominated by intense fluxes of high-energy protons, while the outer belt consists of energetic electrons and ring current ions. However, energetic electrons are also present in the inner belt, which is one of the most difficult places to make clean electron measurements, because of the highly penetrating protons present there. Satellites that traverse the inner radiation belt, defined here as $L^{*}<2.5$, have great difficulty distinguishing signals generated by the electrons from those generated by protons [Vampola, 1998]. The energies of the protons are sufficiently high that they penetrate most shielding and deposit unwanted energy in detectors targeted at electrons. Many of the very early observations were also contaminated by electrons injected into the inner zone regions by the high-altitude nuclear detonation events such as Starfish [Hess, 1963a, 1963b]. The Starfish fluxes slowly decayed at some $L^{*}$ values and more rapidly for $L^{*}>1.7$ or so [Pfitzer and Winckler, 1968]. Prior to Starfish, there were only sparse observations of the natural electron populations in the inner zone [Pizzella et al., 1962; Pfitzer and Winckler, 1968; Hess, 1968, pp. 147-151], and it was not certain that there were MeV electrons present. To compound this situation, there have been relatively few recent satellite missions (within the last 30 years) that covered this region close to the magnetic equator, where the particle fluxes are the highest and one can measure the complete electron pitch angle distribution. One such mission was CRRES (Combined Release and Radiation Effects Satellite), launched more than 24 years ago, and since then, there has been a lack of clean comprehensive electron measurements in this near-equatorial inner magnetosphere region. With the launch of the Van Allen Probes in August 2012 [Mauk et al., 2013] into $\sim 600 \times 30500 \mathrm{~km}$ orbits with $\sim 10^{\circ}$ inclination, we are revisiting this region once more with quality instrumentation.

There has been much discussion about the sources of the inner zone electrons, starting with the possibility that they come from the same neutron albedo process that generates the core inner zone protons [Lenchek et al., 1961; Selesnick et al., 2007, and references therein]. The neutron albedo process was shown early to be too weak to account for the observed electron flux levels, although it predicted that the fluxes would be dominated by $<$ MeV energies. Hess [1968] summarized the limited existing inner zone pre-Starfish event results as showing that the "...spectrum is flat for $100<E e<400 \mathrm{keV}$ " and "... not certain whether there are any electrons present of $E>800 \mathrm{keV}$." More recently, Abel et al. [1994, 1997] presented spectra of equatorially 


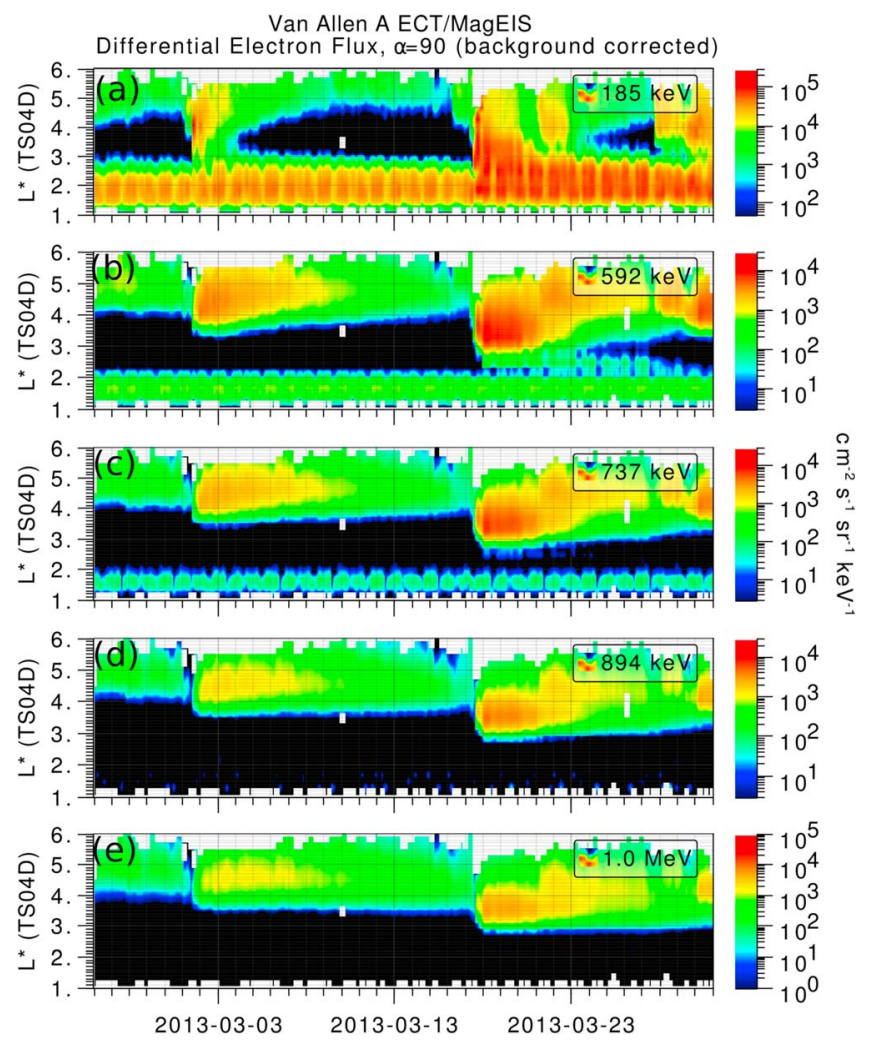

Figure 1. $L^{*}$ versus time spectrograms of MagEIS electron fluxes from five selected energies taken by Van Allen Probe A during 24 February to 31 March 2013.

while Probe $A$ was traversing the inner radiation zone and lower slot region which we define, using Roederer's $L^{*}$ [Roederer, 1970], as $1.2 \leq L^{*} \leq 2.5$, based on the OP77Q [Olson and Pfitzer, 1977] and TS04D [Tsyganenko and Sitnov, 2005] field models. At the low near-equatorial altitudes studied during the quiet 24 February to 1 March 2014 period chosen, both models give essentially the same results for $L^{*}$ and $B / B_{0}$ along the satellite trajectory. We selected traversals through this region that were very close to the magnetic equator with $B / B_{0} \leq 1.025$ in the intervals used. The locally mirroring electrons had second invariant values $K$ such that $0 \leq K<0.01 R_{E} \sqrt{ } \mathrm{G}$. The electron measurements were made by the Magnetic Electron Ion Spectrometers (MagEIS) [Blake et al., 2013] that are part of the Energetic Particle, Composition, and Thermal Plasma (ECT) suite [Spence et al., 2013] on the Van Allen Probes. The three different MagEIS spectrometers relevant to this study are the LOW, MED75, and HIGH units which cover the energy ranges 36-215 keV, 230-1050 keV, and 841-4120 keV, respectively. All three units are mounted on the spacecraft with their fields of view oriented at $105^{\circ}$ to the approximately Sun-pointing spin axis. The data from each spectrometer are accumulated over many angular sectors during each satellite rotation to obtain pitch angle distributions. The MagEIS data used have been corrected by removing any proton response using the algorithm described in S. G. Claudepierre et al. (A background correction algorithm for Van Allen Probes MagEIS electron flux measurements, J. Geophys. Res., in review, 2015). As noted there, not all MagEIS electron channels can be corrected. From those that could, we selected 19 differential MagEIS channels with central energies in the range of 37 to $4080 \mathrm{keV}$ for this study. In addition, the as flown MagEIS HIGH energy channel definitions were revised based on drift-echo analyses [O'Brien et al., 2015] and GEANT4 [Allison et al., 2006] simulations.

\section{Observations}

Figure 1 provides an overview of MagElS electron observations during the late February and March 2013. Figures 1a-1e show the corrected electron fluxes versus $L^{*}$ and time for five selected energies. One can 

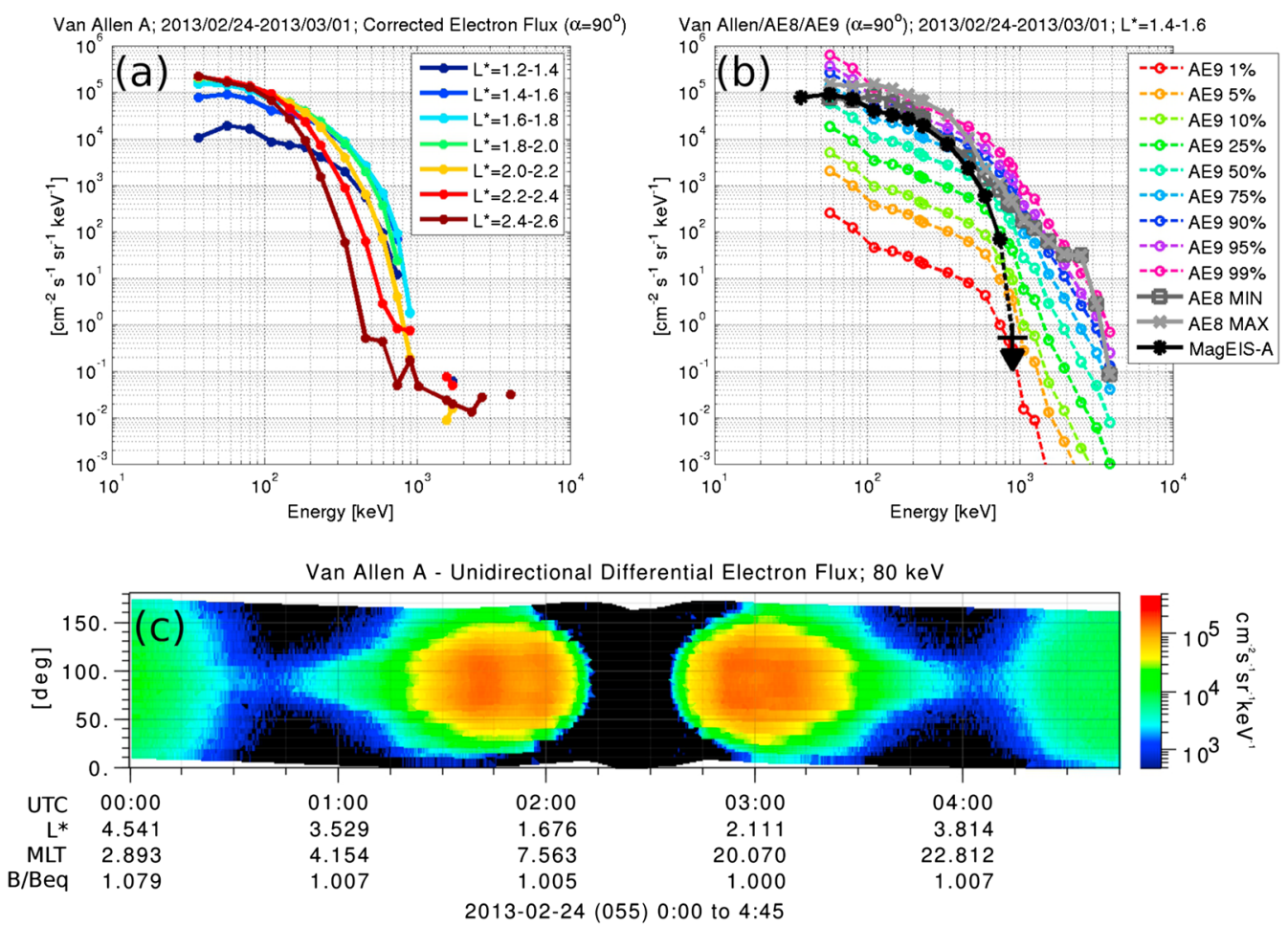

Figure 2. (a) MagEIS equatorially mirroring electron spectra for seven different $L^{*}$ values. (b) Comparison of the $L^{*}=1.4-1.6$ MagEIS spectrum to the different percentile spectra from AE9 and the AE8 MIN and MAX spectra. (c) Pitch angle spectrogram of the $80 \mathrm{keV}$ electron fluxes that were taken near the magnetic equator for $L^{*}<2.5$.

clearly see that only Figures $1 \mathrm{a}-1 \mathrm{c}$ show the electron fluxes above background for $L^{*}<3$. Figure 1 also shows the radiation belt response to the corotating interaction region-driven event on 1 March and the coronal mass ejection-driven event on 17 March [Baker et al., 2014]. The low-energy electrons were transported across the slot region to the inner zone during both storms. This is clearly seen for the $185 \mathrm{keV}$ electrons in Figure $1 \mathrm{a}$. For both the 1 and 17 March events, the electron fluxes $\leq 200 \mathrm{keV}$ were enhanced down to $L^{*}=1.3$. We note that the effect from 1 March is hard to tell on the color spectrogram, but the expansion of the orange-red profile from $L^{*}<2.5$ before to $L^{*}>2.5$ after 1 March is clear. See the supporting information for more details. The inward transport of $592 \mathrm{keV}$ electrons is clear in Figure $1 \mathrm{~b}$ after $17 \mathrm{March}$. The $\sim 737 \mathrm{keV}$ electrons only dipped slightly below $L^{*}=2.75$ following the 17 March event, and the slot region $\left(2 \leq L^{*} \leq 3\right)$ remained essentially empty of $\geq 894 \mathrm{keV}$ electrons during the whole period of Figure 1 . The MagEIS sensors on Van Allen Probe $B$ observed the same features (see supporting information).

We used the quiet period from 24 February to 1 March to accumulate the average electron fluxes in the inner zone and lower slot region. This period was at the end of a long period of relatively quiet time following the activity that occurred on 26 January (not shown). We carefully used only those Van Allen Probe orbits that traversed the $L^{*} \leq 2.5$ region on trajectories that essentially remained at the magnetic equator. We then selected only the $90^{\circ}$ pitch angle fluxes to generate the data shown in Figures $2 \mathrm{a}$ and $2 \mathrm{~b}$. Figure $2 \mathrm{c}$ shows the pitch angle distribution of $80 \mathrm{keV}$ electrons during a perigee pass starting at $L^{*}=4.54$ at 00:00 UT on 24 February moving inbound, traversing the slot and inner zone regions twice, and then continuing outbound beyond $L^{*}=4.5$. Note the low values of $B / B_{0}$ for $L^{*}<2.5$ labeled at the bottom of Figure $2 c$. The corrected data shown have no residual fluxes at small pitch angles in the inner zone region. Such residual fluxes are normally observed when penetrating protons are present, causing a more or less isotropic response in the uncorrected data (see supporting information). Figure $2 \mathrm{c}$ shows the success of the background subtraction algorithm, referenced above, in removing the penetrating protons from the electron data. These corrected data were used to obtain the electron spectra used throughout this paper.

Figure 2a shows the spectra of equatorially mirroring electrons for seven $L^{*}$ bins, each $0.2 \operatorname{Re}$ wide. All the spectra are fairly steeply falling for energies above $400 \mathrm{keV}$ and flatten below $100 \mathrm{keV}$ with the minimum 

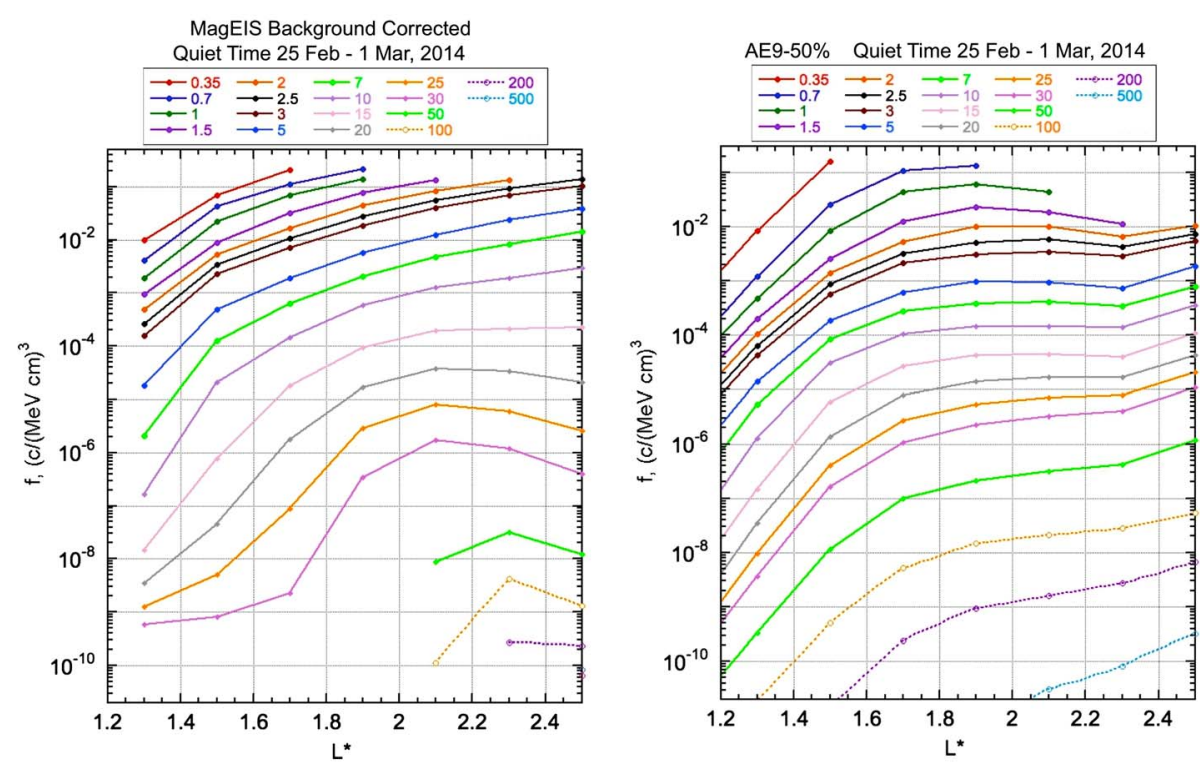

Figure 3. Phase space density $(f)$ versus $L^{*}$ radial profiles for several values of the first adiabatic invariant in MeV/G. (a) The radial profiles observed by MagEIS. (b) The radial profiles from the AE9 quiet time average electron fluxes.

fluxes, at the highest energies, being on the order of $0.1 \mathrm{el} /\left(\mathrm{cm}^{2} \mathrm{~s} \mathrm{sr} \mathrm{keV}\right)$ depending on the $L^{*}$ value. Only data above background are shown. We should note that except for the $L^{*} \geq 2$ curves, the next energy points beyond the highest energy points shown for $1.2 \leq L^{*} \leq 2$ in each spectrum had a value of 0 , indicating that at $>900 \mathrm{keV}$, the electron flux was on the order of $10^{-1} \mathrm{el} /\left(\mathrm{cm}^{2} \mathrm{~s} \mathrm{sr} \mathrm{keV}\right)$ or less. For $L^{*} \geq 2$, there are points with nonzero values in the $10^{-2}$ to $10^{-1}$ range. We take these points to be representative of the minimum possible flux for those $L^{*}$.

As an example, Figure $2 \mathrm{~b}$ compares the MagEIS inner zone spectrum from the $L^{*}=1.4-1.6$ bin with the AE8 maximum and minimum spectra [Vette, 1991] plus several spectra taken from AE9 v1.2 [Ginet et al., 2013] at different percentiles. This shows that AE8 model fluxes exceed those measured by MagEIS in the 700-900 keV range. In fact, the MagEIS $890 \mathrm{keV}$ point, connected by the dashed line, is only an upper limit estimate [O'Brien and Claudepierre, 2015]. At 60-500 keV, the MagEIS flux levels closely match the AE9 75\% curve but are below the AE8 curves. We note that AE9 v2.1 was modified from v1.0 to include a steepening of the electron spectra above $3 \mathrm{MeV}$ in recognition that such high-energy electrons were not consistent with MagEIS observations in low- $L^{*}$ regions. AE-9 v2.1 and v1.0 are essentially the same for energies $\leq 3 \mathrm{MeV}$ at low $L^{*}$, and the $50 \%$ level clearly overestimates the fluxes above $500 \mathrm{keV}$ and underestimates them below $300 \mathrm{keV}$ as shown in Figure $2 b$.

The data from Figure $2 a$ were used to generate phase space densities (PSD) as a function of $L^{*}$ for a range of constant first invariants, $\mu$, as shown in Figure 3a. The PSD was calculated using the prescription in Chen et al. [2005] and are in Geospace Environment Modeling units $(c / M e V / c m)^{3}$, where $c$ is the speed of light. These PSD "radial" profiles are generally rising with increasing $L^{*}$, signaling that the source of the particles is at higher $L^{*}$ values. The $\mu \geq 20$ curves show signs of a flattening or slight peak in PSD near $L^{*}=2$. For comparison, we show an equivalent PSD profile based on the AE9 50\% spectra in Figure 3b. Like Figure 3a, these generally show the rising profiles with increasing $L^{*}$. They also show a flattening of the PSD profile in the same $L^{*} \sim 2$ and $\mu \geq 20$ regions as do the MagEIS data. Careful examination of the MagEIS and AE9 profiles shows that the MagEIS PSD for $\mu \leq 20$ is higher than the AE9 average values. The $\mu=20$ curve is essentially the dividing line between the $L^{*}$ values, where for $\mu \geq 20$, the AE9 PSD are higher than the MagEIS values and smaller $L^{*}$ values with $\mu \mathrm{s}<20$, where the MagEIS PSD values are higher than the AE9 values.

In Figure 4, we compare the MagEIS inner zone fluxes with the published CRRES fluxes [Abel et al., 1994, 1997]. Figure 4a shows a comparison of MagEIS equatorial spectra for $L^{*}=1.35,1.65$, and 1.95 with the CRRES equatorial spectra for $L=1.35,1.65$, and 1.95 (note that $L^{*} \sim L$ for these low values). Clearly, the MagEIS fluxes are lower than the CRRES values, but in both cases, the spectra fall steeply for energies $\geq 500 \mathrm{keV}$ with the 
[a]

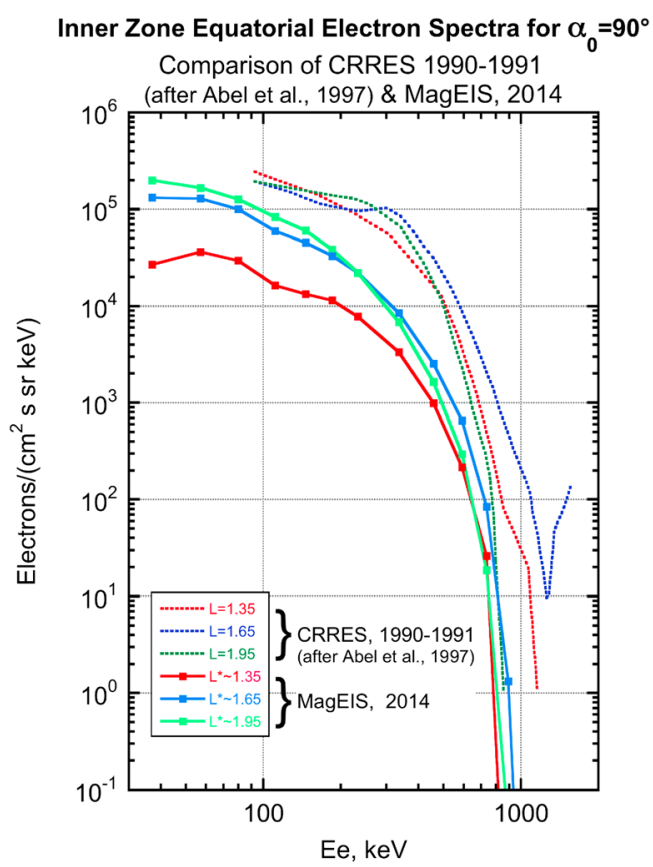

$[b]$
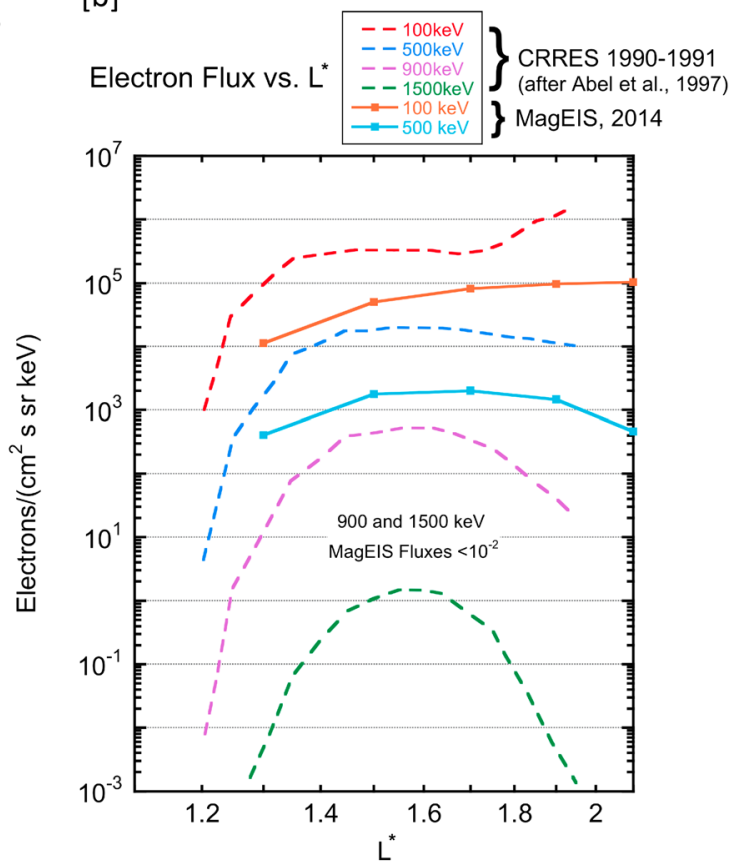

Figure 4. Comparison of (a) MagEIS and CRRES inner radiation zone electron spectra and (b) fluxes versus $L^{*}$. (Note that $L^{*} \sim L$ at the low $L^{*}$ values is shown.)

MagEIS fluxes, orders of magnitude smaller than the CRRES fluxes at 800-900 keV. The MagEIS and CRRES spectral shapes are quite similar. Figure $4 \mathrm{~b}$ shows the electron flux versus $L^{*}(L$ for CRRES) at constant energy. Again, the MagEIS fluxes are much lower than the CRRES fluxes, although the shapes are similar for the $L^{*}$ range covered. The 900 and $1500 \mathrm{keV}$ MagEIS fluxes are at background, as discussed above, so they were not plotted in Figure 4b.

\section{Discussion}

These MagEIS measurements clearly show that there are no significant fluxes, $>0.1 /(\mathrm{cm} 2 \mathrm{~s} \mathrm{sr} \mathrm{keV})$, of MeV electrons in the inner radiation zone and lower edge of the slot region during the period studied. The MagEIS measurements are borne out by Li et al.'s [2015] measurements at much higher energies. The obvious question is whether this is a result of the limited activity levels that occurred during the late 2012 and early 2013 with the result that any $>$ MeV fluxes had decayed away [Baker et al., 2007] or whether this condition existed for the whole time period of the Van Allen Probe observations. As a reference, we note that during the higher activity levels that existed during solar cycle 23, there were a few occasions, prior to 2008, where MeV electrons penetrated into the slot region and inner zone [Fennell et al., 2012a, 2012b; Fennell and Roeder, 2010; Baker et al., 2004]. Limited observations from early Van Allen Probe data taken following the 30 September 2012 storm, during the commissioning phase for the MagEIS sensors, indicated that any electrons present at $\sim 1 \mathrm{MeV}$ were consistent with a background response in MagEIS, as far as we could tell given the preliminary setup of the sensors [Fennell et al., 2012a, 2012b]. However, a more detailed reexamination of those data showed that the $>900 \mathrm{keV}$ channels in both MagEIS M75 and HIGH spectrometers showed no fluxes above the proton-generated background. By 6 October 2012, the MagEIS operational configuration was sufficiently optimized that background subtractions could be made. Since that time and through the period of the current study, there was no sign of $>900 \mathrm{keV}$ electrons, above the MagEIS background, in either the inner zone or lower slot region. A preliminary examination of the data taken from March 2013 to June 2014 shows the same lack of $>900 \mathrm{keV}$ electrons in these regions.

The flattening or peak in the PSD versus $L^{*}$ profiles near $L^{*} \sim 2$ may be related to the peak in the electron flux versus $L$ that Vampola and Gorney [1983] observed. They attributed the peak observed precipitating flux to the effect of transmitters interacting with the electrons in the neighborhood of $L=2$. However, there 
was no mention of a peak at large pitch angles, and one would not be expected from VLF transmitter interactions. This flattening of the PSD profiles also indicates that if radial diffusion [Tomassian et al., 1972] is the quiet time transport process in this region, the process has reached an equilibrium between the cross $L^{*}$ transport rates and the losses from pitch angle diffusion and $d E / d x$ (energy loss per unit distance traveled in the medium) related energy diffusion. For $L^{*}<1.6$, the steepening radial gradients show the effect of relatively rapid atmospheric losses, which dominate the processes there.

At $\leq 500 \mathrm{keV}$, there is considerable evidence that the electron decay rates are sufficiently high that no matter how electrons are injected into regions of $L^{*} \leq 2$, they will have decayed to a more or less equilibrium value within days to weeks [Van Allen, 1966; West and Buck, 1976]. As noted above, there is no evidence of strong injections into the $L \leq 2$ region, with energies $>500 \mathrm{keV}$, during the Van Allen Probe mission up through early March 2013. However, there were injections of $\leq 500 \mathrm{keV}$ electrons observed in multiple cases. As noted above, such injections were observed for both March 2013 storms (see supporting information). These lower energy electron injections into the inner zone and slot region will be discussed in detail in a separate paper.

\section{Conclusions}

The Van Allen Probe MagElS spectrometers do not observe significant fluxes of $\geq$ MeV electrons in the inner radiation zone or bottom side of the electron slot region. It will be important to continue to examine these regions throughout the Van Allen Probe mission to determine whether this lack of MeV electrons is the new normal or whether the mission time period is unique, as might be inferred from solar cycle 23 results noted above. If it is the new normal, then the radiation belt empirical models and the theoretical descriptions of the generation and maintenance of the inner zone electron fluxes will have to be modified to explain this condition.

\section{References}

Abel, B., R. M. Thorne, and A. L. Vampola (1994), Solar cyclic behavior of trapped energetic electrons in Earth's inner radiation belt, J. Geophys. Res., 99(A10), 19,427-19,431, doi:10.1029/94JA01626.

Abel, B., R. M. Thorne, and A. L. Vampola (1997), Energetic electron precipitation from the inner zone, Geophys. Res. Lett, 24, 1983-1986, doi:10.1029/97GL02055.

Allison, J., et al. (2006), Geant4 developments and applications, IEEE Trans. Nucl. Sci., 53, doi:10.1109/TNS.2006.869826.

Baker, D. N., et al. (2004), An extreme distortion of the Van Allen belt arising from the'Hallowe'en' solar storm in 2003, Nature, 432, doi:10.1038/nature03116.

Baker, D. N., S. G. Kanekal, R. B. Horne, N. P. Meredith, and S. A. Glauert (2007), Low-altitude measurements of 2-6 MeV electron trapping lifetimes at $1.5 \leq L \leq 2.5$, Geophys. Res. Lett., 34, L20110, doi:10.1029/2007GL031007.

Baker, D. N., et al. (2014), Gradual diffusion and punctuated phase space density enhancements of highly relativistic electrons: Van Allen Probe observations, Geophys. Res. Lett., 41, 1351-1358, doi:10.1002/2013GL058942.

Blake, J. B., et al. (2013), The Magnetic Electron Ion Spectrometer (MagEIS) instruments aboard the Radiation Belt Storm Probes (RBSP) Spacecraft, Space Sci. Rev., doi:10.1007/s11214-013-9991-8.

Chen, Y., R. H. W. Friedel, G. D. Reeves, T. G. Onsager, and M. F. Thomsen (2005), Multisatellite determination of the relativistic electron phase space density at geosynchronous orbit: Methodology and results during geomagnetically quiet times, J. Geophys. Res., 110, A10210, doi:10.1029/2004JA010895.

Fennell, J. F., and J. L. Roeder (2010), Evolution and energization of energetic electrons in the inner magnetosphere, in The Cluster Active Archive, Astrophysics and Space Science Proceedings, edited by Laakso, et al., pp. 467-489, Springer, Netherlands, doi:10.1007/978-90-481-3499-1_34.

Fennell, J. F., et al. (2012a), Initial look at the electron slot and inner zone: ECT/MagEIS Data, Abstract SM44A-05 presented at 2012 Fall Meeting, AGU, San Francisco, Calif., 3-7 Dec.

Fennell, J. F., S. Kanekal, and J. L. Roeder (2012b), Storm responses of radiation belts during solar cycle 23: HEO satellite observations, in Dynamics of the Earth's Radiation Belts and Inner Magnetosphere, Geophys. Monogr., vol. 199, pp. 3761-384, AGU, Washington, D. C.

Ginet, G. P., et al. (2013), AE9, AP9 and SPM: New models for specifying the trapped energetic particle and space plasma environment, Space Sci. Rev., doi:10.1007/978-1-4899-7433-4_18.

Hess, W. N. (1963a), The artificial radiation belt made on July 9, 1962, J. Geophys. Res., 68(3), 667-683, doi:10.1029/JZ068i003p00667.

Hess, W. N. (Ed) (1963b), Collected papers on the artificial radiation belt from the 9 July 1962 nuclear detonation, J. Geophys. Res., 68, 605-758, doi:10.1029/JZO68i003p00605.

Hess, W. N. (1968), The Radiation Belt and Magnetosphere, pp. 147-151, Blasdell Publ. Co., Waltham, Mass.

Lenchek, A. M., S. F. Singer, and R. C. Wentworth (1961), Geomagnetically trapped electrons from cosmic ray albedo neutrons, J. Geophys. Res., 66(12), 4027-4046, doi:10.1029/JZ066i012p04027.

Li, X., et al. (2015), Upper limit on the inner radiation belt MeV electron intensity, J. Geophys. Res. Space Physics, doi:10.1002/2014JA020777.

Mauk, B. H., N. J. Fox, S. G. Kanekal, R. L. Kessel, D. G. Sibeck, and A. Ukhorskiy (2013), Science objectives and rationale for the Radiation Belt Storm Probe mission, Space Sci. Rev., doi:10.1007/s11214-012-9908-y.

O'Brien, T. P., and S. G. Claudepierre (2015), Fully Bayesian-Poisson calculation of electron foreground count Rate for the MagEIS instrument The Aerospace Corporation, ATR-2015-00906.

O'Brien, T. P., et al. (2015), On the use of drift echoes to characterize on-orbit sensor discrepancies, J. Geophys. Res. Space Physics, doi:10.1002/ 2014JA020859. 
Olson, W. P., and K. A. Pfitzer (1977), Magnetospheric magnetic field modeling, annual scientific report, Air Force Off. of Sci. Res. Contract F44620-75-C-0033, 96 pp., McDonnell Douglas Astronautics Co., Huntington Beach, Calif.

Pfitzer, K. A., and J. R. Winckler (1968), Experimental observation of a large addition to the electron inner radiation belt after a solar flare event, J. Geophys. Res., 73, 5792-5797, doi:10.1029/JA073i017p05792.

Pizzella, G., C. D. Laughlin, and B. J. O'Brien (1962), Note on the electron spectrum in the inner Van Allen belt, J. Geophys. Res., 67(9), 3281-3287, doi:10.1029/JZ067i009p03281.

Roederer, J. G., (1970), Dynamics of Geomagnetically Trapped Radiation, Springer, New York.

Selesnick, R. S., M. D. Looper, and R. A. Mewaldt (2007), A theoretical model of the inner proton radiation belt, Space Weather, 5, S04003, doi:10.1029/2006SW000275.

Spence, H. E., et al. (2013), Science goals and overview of the Energetic Particle, Composition, and Thermal Plasma (ECT) Suite on NASA's Radiation Belt Storm Probes (RBSP) mission, Space Sci. Rev., doi:10.1007/s11214-013-0007-5.

Tomassian, A. D., T. A. Farley, and A. L. Vampola (1972), Inner-zone energetic-electron repopulation by radial diffusion, J. Geophys. Res., 77(19), 3441-3454, doi:10.1029/JA077i019p03441.

Tsyganenko, N. A., and M. I. Sitnov (2005), Modeling the dynamics of the inner magnetosphere during strong geomagnetic storms, J. Geophys. Res., 110, A03208, doi:10.1029/2004JA010798.

Vampola, A. L. (1998), Measuring energetic electrons: What works and what doesn't?, in Measurement Techniques in Space Plasmas: Particles, edited by R. F. Pfaff, J. E. Borovsky, and D. T. Young, AGU, Washington, D. C, doi:10.1029/GM102p0339.

Vampola, A. L., and D. J. Gorney (1983), Electron energy deposition in the middle atmosphere, J. Geophys. Res., 88(A8), 6267-6274, doi:10.1029/JA088iA08p06267.

Vampola, A. L., J. V. Osborn, and B. M. Johnson (1992), CRRES Magnetic Electron Spectrometer AFGL-701-5A (MEA), J. Spacecraft Rockets, 29, 592-595.

Van Allen, J. A. (1966), Some general aspects of geomagnetically trapped radiation, in Radiation Trapped in Earth's Magnetic Field, Astrophys. Space Sci. Libr., vol. 5, edited by B. M. McCormac, pp. 65-75, D. Reidel Publ., Dordrecht, Holland.

Vette, J. I. (1991), The AE-8 trapped electron model environment, National Space Science Data Center (NSSDC) World Data Center "A" for Rockets and Satellites (WDC-A-R\&S), NSDC/WDC-A-R\&S 91-24.

West, H. I., Jr., and R. M. Buck (1976), Energetic electrons in the inner belt in 1968, Planet. Space Sci., 24, doi:10.1016/0032-0633(76)90032-5. 